\title{
Division Theorems in Spaces of Entire Functions with Growth Conditions and their Applications to PDE of Infinite Order*
}

\author{
By \\ Carlos A. BERENSTEIN**, Roger GAY*** and Alekos VIDRAS ****
}

\begin{abstract}
It is shown that every entire function $f \in \operatorname{Exp}_{0}\left(\mathbf{C}^{n}\right), f(0) \neq 0$ is "slowly decreasing". As an application of this property, a theorem on analytic continuation of solutions of infinite order differential equations with constant coefficients is proven.
\end{abstract}

\section{§o. Introduction}

In 1969 C. O. Kiselman [12] proved the following theorem: Given an open, convex set $U \subset \mathbf{C}^{n}$, any holomorphic solution $u$ in $U$ of the linear partial differential equation with constant coefficients $P(D) u=\sum_{|\alpha| \leq m} a_{\alpha} D^{\alpha} u=0$, can be analytically continued to an maximal open convex set $V$, which is independent of $u$ but which depends on the zero set of the principal part $P_{m}$ of $P(z)$ :

$$
P_{m}(z)=\sum_{|\alpha|=m} a_{\alpha} z^{\alpha} .
$$

The method used was a division theorem of certain entire functions by the polynomial $P$ and the following fundamental property:

The distance of any point $z \in \mathbf{C}^{n}$ such that $|P(z)| \leq 1$

to the set $Z_{P}=\left\{z \in \mathbf{C}^{n}: P(z)=0\right\}$ is bounded.

A. Sebbar [15] proved the same type of analytic continuation theorem in the case of infinite order differential operators with constant coefficients. He considered operators of the form $f(D)=\sum_{\alpha \in \mathbf{N}^{n}} a_{\alpha} D^{\alpha}$ so that $f(z)=\sum_{\alpha \in \mathbf{N}^{n}} a_{\alpha} z^{\alpha} \in \operatorname{Exp}_{0}$, the space of entire

* Research partially supported by NSF grants DMS92-25043 and CDR 88-03012.

Communicated by T.Kawai, February 10, 1993. Revised August 3, 1993 and September 3,1 993.

1991 Mathematics Subject classifications: 32A15(35A20)

** University of Maryland, College Park, MD, USA.

*** University of Bordeaux I, Talence, France.

**** RIMS, Kyoto University, Kyoto 606, Japan. Present address: University of Bordeaux I, 3405 Talence, France. 
functions of exponential type zero (that is, for $\forall \varepsilon>0$ there exists a positive constant $C_{\varepsilon}$ so that $\left.|f(z)| \leq C_{\varepsilon} e^{\varepsilon|z|}\right)$. In this case, again, the crucial step was a division theorem in the "correct" space of entire functions. However, since there is no analogue of ( $\mathscr{Q})$ in this case, he restricted himself to the class of operators satisfying the following condition:

$$
\begin{aligned}
& f(z)=\sum_{\alpha \in \mathbb{N}^{n}} a_{\alpha} z^{\alpha} \text { is of exponential type zero and } \forall \varepsilon>0 \text { exists } R_{\varepsilon}>0 \\
& \text { so that } \forall|z|>R_{\varepsilon} \text { and }|f(z)|<1 \text { we have } d\left(z, Z_{f}\right) \leq \varepsilon|z| .
\end{aligned}
$$

An example, due to C.O. Kiselman, shows that not all functions in $\operatorname{Exp}_{0}$ have this property. Very recently, T. Aoki [3] using very deep tools from microlocal analysis, in particular the theory of hyperbolic microdifferential operators of Kashiwara-Shapira [10], proved A. Sebbar's analytic continuation theorem without the assumption ( $\mathscr{P}$ ). More precisely, T. Aoki proves a more general local continuation theorem for the variable coefficient case and then as a corollary obtains the stronger version of the Sebbar's result. One of the important ingredients of the symbol calculus of microdifferential operators used in Aoki's work is a lemma of T. Kawai [11], that allows to define the characteristic set of these operators. This lemma depends on the minimum modulus theorem for holomorphic functions.

In the present work, we give an elementary complex analytic proof of the AokiSebbar theorem. Our approach is in the spirit of Kiselman-Sebbar, through a division theorem in the space of entire functions with growth conditions. By constructing a tube around the set $Z_{f}=\left\{z \in \mathbf{C}^{n}: f(z)=0\right\}$, whose size changes asymptotically, we show that the property $(\mathscr{P})$ is redundant. Our proof of the division theorem has two main ingredients: the minimum modulus principle and inter polation (inspired by [5], see also [11] for similar approach). Furthermore, as an application of the same technique we are able to get the division formula even for the case where the symbols of the infinite order differential operators have coefficients holo morphically dependent on a parameter.

We recall some notation and basic definitions. By $\mathbb{C}^{n}, n \geq 1$, we will denote the space of n-tuples $z=\left(z_{1}, \ldots, z_{n}\right), z_{l} \in \mathbf{C}$, equipped with the norm $|z|=\left(\sum_{i=1}^{n} z_{l} \overline{z_{l}}\right)^{\frac{1}{2}}$. The bracket $\langle$,$\rangle will denote the bilinear product of two elements in \mathbf{C}^{n}$ given by $\langle z, w\rangle=\sum_{i=1}^{n} z_{l} w_{i}$.

Definition 0.1. An entire function $f: \mathbf{C}^{n} \rightarrow \mathbf{C}$ given by $f(z)=\sum_{\alpha \in \mathbf{N}^{n}} a_{\alpha} z^{\alpha}$ is of exponential type zero (infraexponential type) if and only if for every $\varepsilon>0$ there exists $C_{\varepsilon}$ such that for every $z \in \mathbb{C}^{n}$ we have $|f(z)| \leq C_{\varepsilon} \exp (\varepsilon|z|)$.

Let $0 \in \mathbf{C}^{n}$ and $\mathscr{O}=\bigcup_{k \geq 1} H(B(0,1 / k))$ be the space of germs of holomorphic functions near the origin. We know that every operator $L: \mathscr{O} \rightarrow \mathscr{O}$ is continuous if and 
only if $L \mid H(B(0,1 / k))$ is continuous for every $k$. We will be interested in a special class of continuous operators. Assume that we have a function $\Phi(\zeta)=\Sigma_{\alpha} a_{\alpha} \zeta^{\alpha}$, $\alpha \in \mathbf{N}^{n}, \zeta \in \mathbf{C}^{n}$, of exponential type zero. Then we can define

$$
\Phi(D)=\sum_{\alpha} a_{\alpha} D^{\alpha} \delta: \mathscr{O} \rightarrow \mathscr{\mathscr { O }}
$$

a continuous operator (homomorphism), which in turn gives an element of the dual space $\mathscr{O}^{*}$

or, more formally,

$$
\Phi(D)(f): f \in \mathscr{O} \mapsto \sum_{\alpha} a_{\alpha} f^{\alpha}(0) \in \mathbf{C},
$$

$$
\Phi(D)(f): f \in \mathscr{O} \mapsto \sum_{\alpha} a_{\alpha} f^{\alpha}(z) \in \mathbf{C},
$$

for $z$ close enough to the origin. $\Phi(D)$ is an infinite order differential operator with constant coefficients and the function $\Phi$ is called the symbol of infinite order differential operator. Such an infinite order differential operator $L$ acts from $H(\Omega)$ into itself ( $\Omega$ open subset of $\mathbf{C}^{n}$ ). The problem is to study ker $L$, i.e., to determine which holomorphic functions satisfy an infinite order differential equation. This type of problem of considered originally by Ehrenpreis and Martineau in one variable.

Let $\mathscr{U}\left(\mathbf{C}^{n}\right)$ be the set of all convex domains in $\mathbf{C}^{n}$ and for any domain $U$ in $\mathbf{C}^{n}$ let $\mathscr{K}(U)$ be the set of all nonempty, compact convex subsets of $U$. If $A$ is any nonempty subset of $\mathbf{C}^{n}$, then for any $K \in \mathscr{K}\left(\mathbf{C}^{n}\right)$ and for any domain $U \in \mathscr{U}\left(\mathbf{C}^{n}\right)$ we consider the closed sets

$$
\gamma_{A}(K)=\bigcap_{\zeta \in A}\left\{z \in \mathbf{C}^{n}, \operatorname{Re}\langle z, \zeta\rangle \leq H_{K}(\zeta)\right\}
$$

where $H_{K}(\zeta)=\sup _{z \in K} \operatorname{Re}\langle z, \zeta\rangle$. Then we set

$$
\Gamma_{A}(U)=\bigcup_{K \in \mathscr{H}(U)} \gamma_{A}(K)^{o}
$$

We recall that by cone we mean subset of $\mathbf{C}^{n}$ which remains stable under the multiplication by positive constants. Let $A$ be a closed cone in $\mathbf{C}^{n}$, and let $K$ be a convex compact set in an open convex set $\Omega \subset \mathbf{C}^{n}$. Then the set $\Gamma_{A}(\Omega)$ is called the polar enve lope of $\Omega$ with respect to $A$.

Definition 0.2. Let $A$ be an unbounded subset of $\mathbf{C}^{n}$, the an asymptotic cone of $A$, denoted by $\alpha(A)$, is the closed real cone generated by the origin in $\mathbf{C}^{n}$ and by the accumulation points of all the sequences $\left\{\frac{\zeta_{J}}{\left|\zeta_{J}\right|}\right\}_{J \geq 1}, \zeta_{J} \in A$ and $\lim _{J \rightarrow+\infty}\left|\zeta_{J}\right|=+\infty$. 
Example 0.3. Consider $\Phi: \mathbb{C} \rightarrow \mathbb{C}$ defined by $\Phi(z)=\prod_{i=1}^{\infty}\left(1-\frac{z}{n^{3}}\right)$. Then the set of zeroes $Z_{\Phi}$ of $\Phi$, is an unbounded set and $\alpha\left(Z_{\Phi}\right)=\mathbb{R}^{+}$. If $U=\{z:|z|<1\}$ we deduce that

$$
\begin{aligned}
\Gamma_{\alpha\left(Z_{\Phi}\right)}(U) & =\bigcup_{K \in \mathscr{H}(U)}\left(\bigcap_{\zeta \in \alpha\left(Z_{\Phi}\right)}\left\{z \in \mathbf{C}: \operatorname{Re}\langle z, \zeta\rangle \leq H_{K}(\zeta)\right\}\right) \\
& =\{z \in \mathbb{C}: \operatorname{Re} z<1\} .
\end{aligned}
$$

We will need some further notation: for $L$ a differential operator of infinite order with constant coefficients, whose symbol is $\Phi$, the characteristic set $C h(L)$ of $L$, is $C h(L)=\alpha\left(\left\{\zeta \in \mathbb{C}^{n}: \Phi(\zeta)=0\right\}\right)$.

If $A$ is any, non empty subset of $\mathbb{C}^{n}$, we define for any $K \in \mathscr{K}\left(\mathbb{C}^{n}\right)$ and for any $r \geq 0$ :

$$
\begin{aligned}
& \Theta_{A, r}(K)=\bigcap_{\zeta \in A}\left\{z \in \mathbb{C}^{n}, \operatorname{Re}\langle z, \zeta\rangle \leq H_{K}(\zeta)+r\right\} \\
& \Theta_{A}(K)=\overline{U_{r \geq 0} \Theta_{A, r}(K)}
\end{aligned}
$$

For $\mathscr{U}\left(\mathbb{C}^{n}\right)$ we set

$$
\Theta_{A}(\mathscr{U})=\bigcup_{K \in \dddot{R}\left(\mathbf{C}^{n}\right)}\left(\Theta_{A}(K)\right)^{o} .
$$

We will also be using the following

Proposition 3.3 ([12]). If $A$ is an unbounded subset of $\mathbb{C}^{n}$ then for any open convex subset of $\mathbb{C}^{n}$ :

$$
\Gamma_{\alpha(A)}(U)=\Theta_{A}(U) .
$$

We want to give here a simple proof of the following

Theorem (A. Sebbar-T. Aoki). Let L be a differential operator of infinite order with constant coefficients. Let $\Omega$ be an open convex set in $\mathbb{C}^{n}$ and $\Omega^{\prime}$ be the polar envelope of $\Omega$ with respect to the characteristic set $C h(L)$ of $L$. Suppose that $u$ is holomorphic in $\Omega$ and Lu has an analytic continuation to $\Omega^{\prime}$. Then $u$ has analytic continuation to $\Omega^{\prime}$.

Example 0.4. Let $\Omega=U=\{z \in \mathbf{C} ;|z|<1\}$ and $\Phi(D)$ be as in the above Example 0.3 . Then the theorem claims that any holomorphic solution $u$ of $\Phi(D) u=0$ on $U$ can be continued to the half-plane $\{z \in \mathbb{C}: \operatorname{Re} z<1\}$.

\section{§1. Division Theorem}

Theorem 1.1. Let $f$ be an entire function $\mathbf{C}^{n} \rightarrow \mathbf{C}$ of exponential type zero. Let $K \subset L$ be two compact convex subsets of $\mathbf{C}^{n}$ satisfying the following condition: 


$$
H_{L}(z) \leq H_{K}(z)+b \log (2+|z|) \text { whenever } f(z)=0,
$$

for some $b>0$. Assume also that $f(0)=1$. Then, for every entire function $f_{1}$ satisfying:

$$
\log \left|f_{1}(z)\right| \leq H_{L}(z)+a_{1} \log (2+|z|), z \in \mathbf{C}^{n}
$$

and for every $\varepsilon>0$, there exist two entire functions $f_{2}, f_{3}$, depending on $\varepsilon$, such that $f_{1}=f f_{2}+f_{3}$ and two positive constants $a_{2}(\varepsilon)$ and $a_{3}(\varepsilon)$ such that for every $z \in \mathbf{C}^{n}$ :

$$
\begin{aligned}
& \log \left|f_{2}(z)\right| \leq H_{L(\varepsilon)}(z)+a_{2}(\varepsilon) \log (2+|z|) \\
& \log \left|f_{3}(z)\right| \leq H_{K(\varepsilon)}(z)+a_{3}(\varepsilon) \log (2+|z|)
\end{aligned}
$$

where $L(\varepsilon), K(\varepsilon)$ are $\varepsilon$-neighborhoods of $L, K$. The constants $a_{2}(\varepsilon)$ and $a_{3}(\varepsilon)$ depend on $\varepsilon, f, K$ and $L, a_{1}$ and $b$, respectively but not on $f_{1}$.

The proof of the theorem consists of two steps. The first one is to construct a fixed "tube" $T$ around the set

$$
Z_{f}=\left\{z \in \mathbf{C}^{n}: f(z)=0\right\}
$$

with the property for any $\varepsilon>0$ there exists $R_{\varepsilon}$ such that

$$
\forall z \in T \text { with }|z| \geq R_{\varepsilon} d\left(z, Z_{f}\right) \leq 2 \varepsilon|z| .
$$

The second step is the actual proof of the theorem.

\section{Construction of the tube}

Let $\mathscr{L}$ be the family of all complex lines through the origin of $\mathbf{C}^{n}$. If $L$ belongs to $\mathscr{L}$, we can choose $z \in \mathbf{C}^{n},|z|=1$ satisfying $L=L_{z}=\{\lambda z, \lambda \in \mathbf{C}\}$. Then $\lambda \rightarrow f(\lambda z)$ is an entire function of single complex variable $\lambda$ and of exponential type zero. In that manner we reduce the problem from $f: \mathbf{C}^{n} \rightarrow \mathbf{C}$ to a problem of a family of entire functions $f_{z}(\lambda)=f(\lambda z): \mathbf{C} \rightarrow \mathbf{C}$, of exponential type zero. The constants associated to the family are uniform and depend only on $\varepsilon$. That is $\left|f_{\xi}(\lambda)\right| \leq C_{\varepsilon} e^{\varepsilon|\lambda|}$ and the constants $C_{\varepsilon}$ are independent of $z$.

The following lemma is immediate from the usual minimum modulus theorem [14].

Lemma 1.2. Let $\left\{f_{J}\right\}_{j \in J}$ be family of entire functions of growth $(\rho, 0)$ satisfying the following conditions:

(i) $f_{J}(0)=1 \forall j \in J$

(ii) $\forall \varepsilon>0 \exists C_{\varepsilon}$ such that $\left|f_{,}(z)\right| \leq C_{\varepsilon} \exp \left(\varepsilon|z|^{\rho}\right)$ for all $z \in \mathbf{C}$ and all $j$. 
Let $\varepsilon$ and $\eta$ be any given positive numbers with $\eta \leq \frac{3}{2} e$. Let $R<0$, then, inside the disk $|z| \leq R$, but outside a finite family of disks (depending on $j$ ) the sum of whose radii is not greater than $4 \eta R$ (this is uniform), we have:

$$
\log \left|f_{j}(z)\right|>-\frac{D(\eta)}{(2 e)^{\rho}} \log C_{\varepsilon}-D(\eta) \varepsilon R^{\rho},
$$

where

$$
D(\eta)=\left(2+\ln \frac{3 e}{2 \eta}\right)(2 e)^{\rho} \forall j \in J
$$

We will be using the result only in the case $\rho=1$. Note that the size of the exceptional sets is independent of $\varepsilon$. As a consequence of Lemma 1.2 we can prove the following result, that we take from ([4], Lemma 7.4.7).

Lemma 1.3. Let $\left\{f_{J}\right\}_{J \in J}$ be a family of entire functions of exponential type zero, satisfying the condition (i),(ii) of the previous lemma, and let $0<\varepsilon \leq \frac{1}{2}$. There exists $R_{\varepsilon}$ such that for any $j \in J$ and any $z,|z| \geq R_{\varepsilon}$, there are $0<\sigma, \lambda$ satisfying $\frac{\varepsilon}{8}<\lambda<\frac{\varepsilon}{4}, \exp (-2 \varepsilon|z|) \leq \sigma \leq 1$ so that for any $\zeta$ such that $\lambda|z|-\sigma \leq|\zeta-z| \leq \lambda|z|+\sigma$ one has

$$
\left|f_{J}(\zeta)\right| \geq e^{-\varepsilon|\zeta|}
$$

Corollary 1.4. Let $\left\{f_{J}\right\}_{J \in J}$ be as in the previous lemma, $0<\varepsilon \leq \frac{1}{2}$, and let $\mathscr{C}_{\alpha, J}$ be the connected components of the open sets

$$
S\left(f_{j}, \varepsilon\right):=\left\{z \in \mathbf{C}:\left|f_{j}(z)\right|<e^{-\varepsilon|z|}\right\} .
$$

There is $R_{\varepsilon}>0$ such that if $|z| \geq R_{\varepsilon}$ and $z \in \mathscr{O}_{\alpha, J}$, then the diameter of $\mathscr{O}_{\alpha, J}$ can be estimated by

$$
\operatorname{diam} \mathscr{O}_{\alpha, J} \leq \frac{\varepsilon}{2}|z|
$$

Let us now denote by $Z_{J}=\left\{z \in \mathbf{C}: f_{J}(z)=0\right\}$ Assume that a point $z \in \mathbf{C}$ satisfies $|z| \geq R_{\varepsilon}$ and $\operatorname{dist}\left(z, Z_{J}\right) \geq \frac{\varepsilon}{2}|z|$ and $\left|f_{J}(z)\right|<\exp (-\varepsilon|z|)$. We conclude that if $\mathscr{O}_{\alpha, J}$ is the corresponding component of $S\left(f_{J}, \varepsilon\right)$ then $\mathscr{O}_{\alpha, J} \cap S\left(f_{J}, \varepsilon\right)=\emptyset$. Thus, the function $\log \left|f_{J}(\zeta)\right|$ is harmonic in the bounded open set $\mathscr{O}_{\alpha, J}$ and

$$
\log \left|f_{j}(\zeta)\right|=-\varepsilon|\zeta| \text { on } \partial \mathscr{O}_{\alpha, J} .
$$


From (1.3) we conclude that for $\zeta \in \partial \mathscr{O}_{\alpha, j},|\zeta| \leq\left(1+\frac{\varepsilon}{2}\right)|z| \leq \frac{5}{4}|z|$. Hence we have

$$
\log \left|f_{J}(\zeta)\right| \geq-\frac{5}{4} \varepsilon|z| \quad \forall \zeta \in \mathscr{O}_{\alpha, J} .
$$

From the minimum modulus principle for harmonic functions we conclude that

$$
\log \left|f_{J}(z)\right| \geq-\frac{5}{4} \varepsilon|z| \text {. }
$$

This estimate is the main result we need to construct the tube mentioned above. We state it as a proposition with the obvious change of notation.

Proposition 1.5. Let $\left\{f_{J}\right\}_{J \in J}$ be as in Lemma 1.2, $Z_{J}=\left\{z \in \mathbf{C}: f_{J}(z)=0\right\}$, and $0<\varepsilon \leq \frac{1}{2}$. There is $R_{\varepsilon}>0$ such that

$$
\begin{gathered}
\text { if }|z| \geq R_{\varepsilon}>0 \text { and } \operatorname{dist}\left(z, Z_{J}\right) \geq \varepsilon|z| \\
\text { then }\left|f_{J}(z)\right| \geq e^{-e|z|} .
\end{gathered}
$$

Corollary 1.6. Let $f$ be an entire function of exponential type zero in $\mathbf{C}^{n}$ such that $f(0)=1$. If $Z=\left\{z \in \mathbf{C}^{n}: f(z)=0\right\}$ then for any $0<\varepsilon \leq \frac{1}{2}$ there is $R_{\varepsilon}>0$ such that

$$
|z| \geq R_{\varepsilon} \text { and } \operatorname{dist}(z, Z) \geq \varepsilon|z| \text { imply }|f(z)| \geq e^{-\varepsilon|z|} .
$$

Proof. Consider the family $\left\{f_{J}\right\}_{J \in J}$, where $J=S^{2 n-1}=\left\{\zeta \in \mathbf{C}^{n}:|\zeta|=1\right\}$ and $f_{\zeta}(\lambda \zeta), \lambda \in \mathbf{C}$. The last proposition applies to this family. Let $z \in \mathbf{C}^{n},|z| \geq R_{\varepsilon}$, $\operatorname{dist}\left(z, Z_{\jmath}\right) \geq \varepsilon|z|$, and set $\zeta=\frac{z}{|z|}, \lambda=|z|$. It is clear that $\operatorname{dist}\left(z, Z_{\zeta}\right) \geq \operatorname{dist}(z, Z) \geq \varepsilon|\lambda|$, thus

$$
|f(z)|=\left|f_{\zeta}(\lambda)\right| \geq e^{-\varepsilon|\lambda|}=e^{-\varepsilon|z|} .
$$

Let us now define a tube about the variety $Z$ of zeroes of $f$, i.e., a closed set whose interior contains $Z$ as follows, for $\varepsilon=\frac{1}{2^{m}}$, let $R_{m}$ be the value from the Corollary 1.6. We can assume that $R_{m+1} \geq R_{m}+1$.

$$
T:=\bigcup_{m=1}^{\infty}\left\{z \in \mathbf{C}^{n}: \operatorname{dist}(z, Z) \leq \frac{|z|}{2^{m}} \text { and }|z| \leq R_{m}\right\}
$$

It is easy to see that $Z \subset T^{0}$ and $T$ is closed. 
Furthermore, for any $\varepsilon>0$ there is a value $R_{\varepsilon}^{\prime}$ so that $|f(z)| \geq e^{-\varepsilon|z|}$ for any $z \notin T,|z|>R_{\varepsilon}^{\prime}$. In fact, choose $m_{0} \gg>1$ so that $2^{-m_{0}} \leq \varepsilon$ and let $R_{\varepsilon}^{\prime}=R_{m_{0}}$. If $|z|>R_{\varepsilon}^{\prime}$, let $m$ be such that $R_{m} \leq|z| \leq R_{m+1}$. As $z \notin T$ it follows that $\operatorname{dist}(z, Z)>\frac{|z|}{2^{m+1}}$ so that by the choice of $R_{m}$

$$
|f(z)| \geq e^{-\frac{|z|}{2^{m+1}}}>e^{-\varepsilon|z|}
$$

because $m \geq m_{0}$ and thus $2^{-m-1}<\varepsilon$.

In the proof that follows we will also use a neighbourhood $T_{1}$ of $T$ defined as follows. Let $R_{0}=-1$ then

$$
T_{1}=\bigcup_{m=1}^{\infty}\left\{z \in \mathbb{C}^{n}: R_{m-1}<|z| \leq R_{m}, \operatorname{dist}(z, T) \leq e^{-\frac{|z|}{2^{m}}}\right\} .
$$

\section{§2. Proof of the Main Theorem $\mathbb{1} .1$}

From the Whitney lemma [9] we conclude there is a function $\chi \in C^{\infty}\left(\mathbb{C}^{n}\right)$ such that $\chi \equiv 1$ on $T$, and satisfying the estimate:

$$
|\bar{\partial} \chi(z)| \leq \mathscr{K} e^{\frac{|z|}{2^{m}}} \text { if } R_{\mathrm{m}-1}<|z| \leq R_{\mathrm{m}},
$$

for some constant $\mathscr{K}$ and $R_{0}=-1$ as earlier.

Let us now define

$$
f_{2}=\frac{f_{1}(1-\chi)}{f}+v
$$

for $v \in C^{\infty}\left(\mathbb{C}^{n}\right)$ is to be determined. Let also

$$
f_{3}=f_{1} \chi-f v .
$$

We need to show that $\bar{\partial} f_{2}=0$, that is

$$
\begin{gathered}
\bar{\partial} v=-\bar{\partial}\left(\frac{f_{1}(1-\chi)}{f}\right) \\
= \begin{cases}\frac{f_{1} \bar{\partial} \chi}{f} & \text { on } T_{1} / T^{0} \\
0, & \text { otherwise. }\end{cases}
\end{gathered}
$$

Denote by $\omega$ the $\bar{\partial}$-closed $(0,1)$ form defined by the last bracket. Note that because $Z \subset T$ we have that $\omega$ is a smooth form. We proceed now to estimate $|\omega|$. There are three parts to this estimate. The estimate (2.1) takes care of $\bar{\partial} \chi$. The denominator of $\omega, f$, has been studied just after the definition of the tube $T$. Finally, we have to use 
the condition (1.1) to estimate $f_{1}$ on $T_{1}$. For that purpose observe that if $z \in T_{1}, R_{m-1}<|z| \leq R_{m}$, then there is a point $\zeta \in T$ such that

$$
|\zeta-z| \leq e^{-\frac{|z|}{2^{m}}} \leq 1
$$

so that

$$
R_{m-2} \leq R_{m-1}-1 \leq|z|-1 \leq|\zeta| \leq|z|+1 \leq R_{m}+1 \leq R_{m+1} .
$$

Thus from the definition (1.6) of $T$ we have

$$
\operatorname{dist}(\zeta, Z) \leq \frac{|\zeta|}{2^{m-2}}
$$

Let $\eta \in Z$ realizing the distance inequality (2.5) and let $\rho>0$ be such that $L<B(0, \rho)$. It follows that for any $w \in \mathbf{C}^{n}$

$$
\left|H_{K}(w)\right| \leq\left|H_{L}(w)\right| \leq \rho|w| .
$$

Since $H_{L}, H_{K}$ are subadditive

$$
\begin{aligned}
H_{L}(z) & \leq H_{L}(\eta)+\rho|z-\eta| \leq H_{L}(\eta)+\rho \frac{|\zeta|}{2^{m-2}} \\
& \leq H_{L}(\eta)+\rho^{\prime} \frac{|\zeta|}{2^{m}}+\rho^{\prime}
\end{aligned}
$$

and

$$
H_{K}(z) \geq H_{K}(\eta)-\rho^{\prime} \frac{|\zeta|}{2^{m}}-\rho^{\prime}
$$

Using (1.1) we have

$$
H_{L}(z) \leq H_{K}(z)+2 \rho^{\prime} \frac{|\zeta|}{2^{m}}+2 \rho^{\prime}+b^{\prime} \log (2+|z|)
$$

for some $\rho^{\prime}, b^{\prime}>0$. Therefore, there is a constant $a^{\prime}$ such that for $z \in T_{1}$, $R_{m-1}<|z| \leq R_{m}$, one has

$$
\log \left|f_{1}(z)\right| \leq H_{K}(z)+2 \rho^{\prime} \frac{|\zeta|}{2^{m}}+a^{\prime} \log (2+|z|)
$$

As a consequence, for $z \in T_{1} \cap T^{0}, R_{m-1}<|z| \leq R_{m}$ we obtain

$$
|\omega(z)| \leq \mathscr{K} \exp \left(H_{K}(z)+2\left(\rho^{\prime}+1\right) \frac{|\zeta|}{2^{m}}+a^{\prime} \log (2+|z|),\right.
$$


where $\mathscr{K}$ is the constant from (2.1). Let $\varepsilon>0$, choose $m_{0}$ such that for $m \geq m_{0}$ we have $\frac{\rho^{\prime}+1}{2^{m-1}} \leq \varepsilon$ and let $K_{\varepsilon}:=\left\{z \in \mathbf{C}^{n}: \operatorname{dist}(z, K) \leq \varepsilon\right\}$ then

$$
H_{K \varepsilon}(w)=H_{K}(w)+\varepsilon|w| \quad \forall w .
$$

It follows from (2.9), (2.10), and the fact that supp $\omega \subset T_{1} \backslash T^{0}$, that for $|z| \geq R_{m_{0}-1}$

$$
|\omega(z)| \leq \mathscr{K} \exp \left(H_{K_{\varepsilon}}(z)+a^{\prime} \log (2+|z|)\right) .
$$

By the smoothness of $\omega$ we can conclude that there is $a^{\prime \prime}=a^{\prime \prime}(\varepsilon)>0$ so that we have the global estimate

$$
|\omega(z)| \leq \exp \left(H_{K_{\varepsilon}}(z)+a^{\prime \prime} \log (2+|z|)\right) .
$$

Now we are looking for a plurisubharmonic function $\phi$ in $\mathbf{C}^{n}$ with the property that $|\omega(\zeta)|^{2} \exp (-\phi)$ is integrable in $\mathbf{C}^{n}$ with respect to the Lebesgue measure. In other words by using the last inequality one can deduce

$$
|\omega(\zeta)|^{2} \exp (-\phi(\zeta)) \leq \exp \left(2 H_{K_{\varepsilon}}(\zeta)+2 a^{\prime \prime}(\varepsilon) \log (2+|\zeta|)-\phi(\zeta)\right)
$$

The idea is to set

$$
\exp \left(2 H_{K_{\varepsilon}}(\zeta)+2 a^{\prime \prime}(\varepsilon) \log (2+|\zeta|)-\phi(\zeta)\right)=(2+|\zeta|)^{-(2 n+1)}
$$

Then, it is natural to choose

$$
\phi(\zeta)=\phi_{\varepsilon}(\zeta)=2 H_{K_{\varepsilon}}+2 a^{\prime \prime}(\varepsilon) \log (2+|\zeta|)+(2 n+1) \log (2+|\zeta|)
$$

By Hörmander's theorem ( [9], Theor. 4.4.2) there exists function $v \in C^{\infty}\left(\mathbf{C}^{n}\right)$ such that $\bar{\partial} v=\omega$ and

$$
M=\int_{\mathbf{c}^{n}} \frac{\left|v^{2} \exp (-\phi)\right|}{\left(1+|z|^{2}\right)^{2}} d \lambda \leq 1 / 2 \int_{\mathbf{c}^{n}}|\omega(\zeta)|^{2} \exp (-\phi(\zeta)) d \lambda<\infty .
$$

We obtain an estimate for $v$ by means of the following lemma:

Lemma 2.1 ([9]). Let $\Omega$ be an domain in $\mathbf{C}^{n}, Q$ be a compact subset of $\Omega$. Then exists a constant $c$ so that for any function $u \in C^{1}(\Omega)$ :

$$
\sup _{Q}|u| \leq c\left\{\sup |\overline{\partial u}|+\int_{\Omega}|u| d \lambda\right\}
$$

where the integration is with respect to Lebesgue measure.

We apply this lemma taking as $\Omega=B(0,1), Q=\{0\}$ and as $u$ the function $z \rightarrow v(\zeta+z)$ with $\zeta \in \mathbf{C}^{n}$ fixed. We thus have 


$$
|v(\zeta)| \leq c^{\prime}\left\{\sup _{|\theta| \leq 1}|\omega(\zeta+\theta)|+\left(\int_{\Omega}\left|v^{2}\right| d \lambda\right)^{\frac{1}{2}}\right\}
$$

On the other hand

$$
\int_{\Omega}|v(\zeta)|^{2} d \lambda \leq \int_{\mathrm{c}^{n}} \frac{\left|v^{2} \exp (-\phi)\right|}{\left(1+|z|^{2}\right)^{2}} d \lambda \times \sup _{z \in \Omega}\left\{\left(1+|z|^{2}\right)^{2} \exp (\phi(z))\right\}
$$

that is,

$$
\left(\int_{\Omega}|v(\zeta)|^{2} d \lambda\right) \leq \sqrt{M} \sup _{z \in \Omega}\left(1+|z|^{2}\right) \exp \left(\frac{1}{2} \varphi(z)\right)
$$

and

$$
\left|\sup _{|\theta| \leq 1} \omega(\zeta+\theta)\right| \leq \Psi_{\varepsilon} \exp \left(H_{K_{\varepsilon}}(\zeta)+a^{\prime \prime \prime}(\varepsilon) \log (2+|\zeta|)\right.
$$

for a new constant $a^{\prime \prime \prime}=a^{\prime \prime \prime}(\varepsilon)$. Therefore, using (2.12), we obtain some constant $C_{1}(\varepsilon)>0$ such that

$$
\log |v(\zeta)| \leq H_{K_{\varepsilon}}(\zeta)+C_{1}(\varepsilon) \log (2+|\zeta|), \zeta \in \mathbf{C}^{n} .
$$

The last step is to show that $f_{2}$ and $f_{3}$ satisfy the inequalities in the statement of the Theorem 1.1.

$$
\left|f_{2}(\zeta)\right|=\left|\left(\frac{f_{1}(1-\chi)}{f}+v\right)(\zeta)\right| \leq \begin{cases}|v(\zeta)|, & \text { on } T \\ \left|\frac{f_{1}}{f}\right|(\zeta)+|v(\zeta)|, & \text { otherwise. }\end{cases}
$$

On $T^{c}$ we have

$$
\begin{aligned}
\left|\frac{f_{1}}{f}(\zeta)\right|+|v(\zeta)| \leq & C_{2}(\varepsilon) \exp (\varepsilon|\zeta|) \exp \left[H_{L}(\zeta)+a_{1} \log (2+|\zeta|)\right] \\
& +\exp \left[H_{K_{\varepsilon}}(\zeta)+C_{1}(\varepsilon) \log (2+|\zeta|)\right] \\
\leq & C_{2}(\varepsilon) \exp \left[H_{L_{\varepsilon}}(\zeta)+a_{1} \log (2+|\zeta|)\right] \\
& +\exp \left[H_{K_{\varepsilon}}(\zeta)+C_{1}(\varepsilon) \log (2+|\zeta|)\right] \\
\leq & \exp \left[H_{L_{\varepsilon}}(\zeta)+C_{3}(\varepsilon) \log (2+|\zeta|)\right]
\end{aligned}
$$

Combining the above we have

$$
\left|f_{2}(\zeta)\right| \leq \exp \left[H_{L_{\varepsilon}}(\zeta)+a_{2}(\varepsilon) \log (2+|\zeta|)\right]
$$

Also

$$
\left|f_{3}(\zeta)\right|=\left|f_{1}-f v\right|(\zeta) \leq \begin{cases}|f v|, & \text { on } T_{1}^{c} \\ \left|f_{1}\right|+|f v| & \text { on } T_{1}\end{cases}
$$


Hence for any $\zeta \in \mathbb{C}^{n}$ we have

$$
\begin{aligned}
|f v(\zeta)| & \leq C_{2}(\varepsilon) \exp \left(\varepsilon|\zeta|+H_{K_{\varepsilon}}(\zeta)+C_{1}(\varepsilon) \log (2+|\zeta|)\right) \\
& \leq \exp \left(H_{K_{2 \varepsilon}}(\zeta)+C_{3}(\varepsilon) \log (2+|\zeta|)\right)
\end{aligned}
$$

On $T$, we have the following estimate

$$
\left|f_{1}(\zeta)\right| \leq \exp \left[H_{L}(\zeta)+a_{1} \log (2+|\zeta|)\right] \leq \exp \left[H_{K_{\varepsilon}}+\left(a_{1}+b(\varepsilon)\right) \log (2+|\zeta|)\right] .
$$

Combining the above

$$
\left|f_{3}(\zeta)\right| \leq \exp \left[H_{K_{\varepsilon}}(\zeta)+a_{3}(\varepsilon) \log (2+|\zeta|)\right]
$$

This completes the proof of the division theorem.

Actually we can modify slightly the proof using a lemma from [4] (see Lemma 3.4 below) and obtain a decomposition independent of $\varepsilon$, with the same estimates.

\section{§3. Division Theorem with Holomorphic Parameter}

Here we will show that we can derive the same formula for the symbols of infinite order differential operators with coefficients dependent holomorphically on a parame ter $t \in \mathbb{C}^{n}$.

Let $X$ be an open set in $\mathbb{C}^{n}$. An operator $P$, whose coefficients depend holomorphically on a parameter $t$ can be written in the form

$$
P=P(t, D)=\sum_{\alpha} a_{\alpha}(t) D^{\alpha}
$$

with $D=\left(D_{1}, \ldots, D_{n}\right)=\left(\frac{\partial}{\partial x_{1}}, \ldots, \frac{\partial}{\partial x_{n}}\right)$ where $a_{\alpha}$ are holomorphic functions on $X$ satisfying the following estimates: For each compact set $K \subset \subset X$ and for each $h>0$, there exists a constant $C>0$ such that

$$
\sup _{t \in R}\left|a_{\alpha}(t)\right| \leq C h^{|\alpha|} \alpha !^{-1}
$$

for any multi-index $\alpha=\left(\alpha_{1}, \ldots \alpha_{n}\right)$. Here we set $|\alpha|=\alpha_{1}+\ldots+\alpha_{n}, \alpha !=\alpha_{1} ! \ldots \alpha_{n} !$. If there is $m \geq 0$ such that $a_{\alpha} \equiv 0$ for every $\alpha$ satisfying $|\alpha|>m$, we say that $P$ is of fi nite order less or equal to $m$. Otherwise $P$ is said to be of infinite order. If we replace $D$ by $\xi=\left(\xi_{1}, \ldots, \xi_{n}\right) \in \mathbb{C}^{n}$ in $(R 1)$, we have a holomorphic function in $(t, \xi) \in X \times \mathbb{C}^{n}$ :

$$
P(t, \xi)=\sum_{\alpha} a_{\alpha}(t) \xi^{\alpha}
$$


By $(R 2), P(t, \xi)$ is an entire function of exponential type zero in $\xi$, namely, an entire function satisfying the following estimate: For every compact set $K \subset \subset X$ and for every $h>0$, there is a positive constant $C$ such that

$$
\sup _{t \in K}|P(t, \xi)| \leq C \exp (h|\xi|) \text {. }
$$

We call the function $P(t, \xi)$ the symbol of $P$. Note that $C=C(h, K)$. Note that these definitions make sense in a complex manifold $X$.

Assume that $P(t, D), P_{1}(t, D)$ are infinite order differential operators whose coefficients depend holomorphically on a parameter. Let $f(t, \xi), f_{1}(t, \xi)$ be their symbols. Fix $t_{0} \in X$, then $f\left(t_{0}, \xi\right)=f_{t_{0}}(\xi)$ is of exponential type zero in the variable $\xi$. Now we can state and prove the following:

Theorem 3.1. Let $f\left(t_{0}, \xi\right), f_{1}(t, \xi)$ be as above and $f_{1}$ is analytic in t. Assume that there exist $K \subset L$, two compact convex subsets of $\mathbf{C}^{n}$ satisfying the following condition:

$$
H_{L}(\xi) \leq H_{K}(\xi)+o(\xi) \text { whenever } f_{t_{0}}(\xi)=0 .
$$

Assume also that $f_{t_{0}}(0)=f\left(t_{0}, 0\right)=1$. Then for every entire function $f_{1}(t, \xi)=f_{1, t}(\xi)$, satisfying the following uniformity condition for any $t \in X$ :

$$
\log \left|f_{1, t}(\xi)\right| \leq H_{L}(\xi)+o(\xi) \xi \in \mathbf{C}^{n}
$$

and for every $\varepsilon>0$, there exist two entire functions $f_{2, t}(\xi)=f_{2}(t, \xi)$, $f_{3, t}(\xi)=f_{3}(t, \xi)$, which are analytic in $t$, such that

$$
f_{1}(t, \xi)=f\left(t_{0}, \xi\right) f_{2}(t, \xi)+f_{3}(t, \xi)
$$

such that for every $\xi \in \mathbf{C}^{n}$ :

$$
\begin{aligned}
& \log \left|f_{2, t}(\xi)\right| \leq H_{L_{\varepsilon}}(\xi)+o(\xi) \\
& \log \left|f_{3, t}(\xi)\right| \leq H_{K_{\varepsilon}}(\xi)+o(\xi)
\end{aligned}
$$

where $L(\varepsilon), K(\varepsilon)$ are $\varepsilon$-neighbourhoods of $L, K$ respectively.

Note. The uniformity assumption on $\left|f_{1}\right|$ is not unnatural, it holds for every $L$ compact, convex set which contains the origin in the interior. Hence, when this does not occur, we get more refined estimate on the growth of $f_{1}$, and eventually of $f_{2}, f_{3}$. Furthermore, the assumption that $K \subset L$ is not essential, since otherwise we can set as $L$ the convex hull $c v(K \bigcup L) \subset X$ if $X$ is convex. Now we are ready to proceed with the rough sketch of the proof

Proof of the Theorem 3.1. $f\left(t_{0}, \xi\right)=f_{t_{0}}(\xi)$ is of exponential type zero in the variable $\xi$. Construct a tube, as in Theorem 1.1, around the set $Z_{f_{t_{0}}}=\left\{\left(t_{0}, \xi\right)\right.$ 
$\left.\in U \times \mathbf{C}^{n}: f\left(t_{0}, \xi\right)=0\right\}$. Then for any $t \in U$, we will divide the function $f_{1}(t, \xi)$ $=f_{t}(\xi)$ by the same function $f\left(t_{0}, \xi\right)$ as in the previous division theorem, only here we are interested in a estimates of the different type. Define:

$$
f_{2, t}=\frac{f_{1, t}(1-\chi)}{f_{t_{0}}}+v_{t}
$$

where $\chi$ is independent of $t$ and it is the Whitney function corresponding to $t_{0}$ in the construction of the tube, and $v_{t} \in C^{\infty}\left(\mathbf{C}^{n}\right)$ is to be determined.

$$
f_{3, t}=f_{1, t} \chi-f_{t_{0}} v_{t} .
$$

We want to show that $f_{2, t}(\xi)=f_{2}(t, \xi), f_{3, t}(\xi)=f_{3}(t, \xi)$ have the "appropriate growth" in $\xi$ and are holomorphic in $t$. In the same way as before we obtain

$$
\omega_{t}= \begin{cases}\frac{f_{1, t} \overline{\partial \chi}}{f_{t_{0}}} & \text { on } T_{1} \backslash T^{0} \\ 0, & \text { otherwise. }\end{cases}
$$

Next, repeating the argument of the Theorem 1.1, we have the following estimate:

$$
\left|\omega_{t}\right| \leq \mathscr{K}(\varepsilon) \exp (\varepsilon|z|)\left|f_{1, t}\right| .
$$

Again, repeating the argument of the previous theorem we extend the condition $(\mathscr{D})$ to any $\xi \in T_{1}$. Therefore

$$
H_{L}(\xi) \leq H_{K}(\xi)+2 \varepsilon|\xi|\left(a_{K}+a_{L}\right)+o(\xi)
$$

where $a_{K}=\sup _{t \in K}|t|$ and $a_{L}=\sup _{t \in L}|t|$. Finally, we deduce the following:

$$
\begin{aligned}
H_{L}(\xi) & \leq H_{K}(\xi)+\left(a_{K}+a_{L}+1\right) \varepsilon|\xi|+b(\varepsilon) \\
& \leq H_{K}(\xi)+m \varepsilon|\zeta|+b(\varepsilon)
\end{aligned}
$$

where $m=\left[a_{K}+a_{L}\right]+2$ and $b(\varepsilon)$ is chosen so that to take into account those $\xi \in T_{1}$ and $|\xi| \leq R_{\varepsilon}$. We can see that the same estimate hold for the sequence $\varepsilon_{n}=\frac{1}{2^{n}}, n=1$, $2, \ldots$ and as $\varepsilon \downarrow 0$ the constants $\mathscr{K}(\varepsilon) \uparrow+\infty$. Therefore applying the Lemma 3.2 below we can obtain a weight $p(|\xi|)$ so that

$$
\left|\omega_{t}(\xi)\right| \leq e^{H_{K}(\xi)+p(\xi \xi)}, \text { where } \lim _{|\xi| \mapsto 0} \frac{p(|\xi|)}{|\xi|}=0 .
$$

Lemma 3.2 ([4], Lemma 3.5.9 or [7], Proposition 1.7). Let $\left\{B_{n}\right\}_{n \geq 0}$ be an increasing sequence of real positive numbers converging to $+\infty$ so that the 
values $t_{n}=(n+1) \log 2+\log \left(B_{n+1}-B_{n}\right)$ are also increasing and $t_{n+1} \geq t_{n}+(1+\log 2)$. Consider the function:

$$
\Phi(t)=\inf _{n \geq 0}\left\{\frac{1}{2^{n}} e^{t}+B_{n}\right\}, t \geq 0 .
$$

Then there exists a function $f$ positive, convex, increasing over $[0,+\infty)$ so that:

$$
f(t) \leq \Phi(t) \leq 2 f(t) .
$$

Hence

$$
\left|\omega_{t}(\xi)\right|^{2} \leq e^{2 H_{K}(\xi)+2 p(\xi \xi)} .
$$

As in Theorem 1.1 we choose a weight $\phi$ by

$$
\phi\left(|\xi|=2 H_{K}(\xi)+2 p(|\xi|)+(2 n+1) \log (2+|\xi|) .\right.
$$

Again, repeating the steps of the Theorem 1.1, we deduce that there exist a functions $v_{t} \in C^{\infty}\left(\mathbf{C}^{n}\right)$ such that $\bar{\partial} v_{t}=\omega_{t}$ and

$$
M=\int_{\mathbf{C}^{n}} \frac{\left|v_{t}^{2} \exp (-\phi)\right|}{\left(1+|\xi|^{2}\right)^{2}} d \lambda \leq 1 / 2 \int_{\mathbf{C}^{n}}\left|\omega_{t}(\xi)\right|^{2} \exp (-\phi(\xi)) d \lambda \leq \infty .
$$

Furthermore, we obtain the following upper bound for $\log \left|v_{t}\right|$, by using the same type of the argument:

$$
\log \left|v_{t}\right| \leq H_{K}(\xi)+\phi(|\xi|)+\text { const, where } \lim _{|\xi| \mapsto \infty} \frac{\phi(|\xi|)}{|\xi|}=0
$$

We can always assume $\phi \in C^{\infty}$ by convolution with multiplier, if necessary. The last inequality allows us to deduce, by exactly the same argument that $f_{2, t}(\xi)=f_{2}(t, \xi)$, $f_{3, t}(\xi)=f_{3}(t, \xi)$ satisfy the claimed growth conditions in the variable $\xi$. Therefore the only property which remains to verify is that of analyticity in the variable $t$. The argument is simple, but for the sake of completeness, will be given here. The basic tool for that will be elementary Hilbert space theory. Our space will be the completion of $C^{\infty}\left(\mathbf{C}^{n}, \phi\right)$, where the weight $\phi$ was determined above, equipped with the inner product

$$
\langle f, g\rangle_{\phi}=\int_{\mathbf{C}^{n}} f \bar{g} \frac{e^{-\phi}}{\left(1+|\xi|^{2}\right)^{(2 n+1)}} d \xi
$$

Let

$$
\mathscr{H}^{(0,0)}=\left\{f \in C^{\infty}\left(\mathbf{C}^{n}, \phi\right)\right\}
$$

$\mathscr{H}^{(0.1)}=\left\{(0.1)\right.$-forms with $C^{\infty}$ coefficients, $\left.g: \bar{\partial} g=0, g \in L^{2}\left(\mathbf{C}^{n}, \phi\right)\right\}$. 
Observe that if $v_{t} \in C^{\infty}\left(\mathbf{C}^{n}, \phi\right)$ is a solution of $\bar{\partial} v_{t}=\omega_{t}$ then $f+v_{t}$ is also a solution of $\bar{\partial} v_{t}=\omega_{t} \forall f \in \mathscr{H}^{(0,0)}$ so that $\bar{\partial} f=0$. Then by Hörmander's a priori estimates for $\bar{\partial}$, the operator $\bar{\partial}$ is onto from $\mathscr{H}^{(0,0)}$ to $\mathscr{H}^{(0,1)}$. This allows us to define a mapping

$$
P: \mathscr{H}^{(0,1)} \rightarrow \mathscr{H}^{(0,0)} .
$$

This is done as follows, to any element $\omega$ in the space $\mathscr{H}^{(0,1)}$ we make correspond the solution $v$ from the space $\mathscr{H}^{(0,0)}$ so that $\|v\|$ has the minimum norm. We will show that the mapping $P$ is linear. It is a standard argument then that the analyticity of $\omega_{t}$ in $t$ will imply the analyticity of $v_{t}$ in $t$ and, therefore, that of $f_{2}(t, \xi), f_{3}(t, \xi)$ in the parameter $t$. For reasons of simplicity we will prove the linearity for $\omega_{t}$, the general argument being exactly the same.

1) Homogeneity: Let $a \in \mathbf{C}$ and $\bar{\partial} v_{t}=\omega_{t}$. Then, we want to show that $P\left(a \omega_{t}\right)=a P\left(\omega_{t}\right)$. By the definition of $P$ there exists $u$ so that $\bar{\partial} u_{t}=a \omega_{t}$, but also $\bar{\partial}\left(a v_{t}\right)=a \omega_{t}$. Therefore $a v_{t}=u_{t}+h_{t}$, where $h_{t}$ is holomorphic. Therefore, by the minimality of $u_{t},\left\|a v_{t}\right\| \geq\left\|u_{t}\right\|$. But $\frac{1}{a} u_{t}$ is a solution of $\bar{\partial}\left(v_{t}\right)=\omega_{t}$. Thus, by minimality $\left\|\frac{1}{a} u_{t}\right\| \geq\left\|v_{t}\right\|$. Hence, $a v_{t}=u_{t}$. So $P$ is homogeneous.

2) Additivity: Consider the following

$$
\begin{aligned}
& \bar{\partial} v_{t, 1}=\omega_{t, 1}, P\left(\omega_{t, 1}\right)=v_{t, 1}, \\
& \bar{\partial} v_{t, 2}=\omega_{t, 2}, P\left(\omega_{t, 2}\right)=v_{t, 2}, \\
& \bar{\partial} v_{t}=\omega_{t, 1}+\omega_{t, 2}, P\left(\omega_{t, 1}+\omega_{t, 2}\right)=v_{t} .
\end{aligned}
$$

We want to show that $v_{t, 1}+v_{t, 2}=v_{t}$. Since $\mathscr{H}^{(0,0)}$ is a closed subspace of $L^{2}\left(\mathbb{C}^{n}, \phi\right)$, for any $v_{t}$ so that, $\bar{\partial} v_{t}=\omega_{t}$, we have that

$$
\left\|v_{t}-f_{v_{t}}\right\|=\inf _{f \in \mathbb{N}^{(0,0)}}\left\|v_{t}-f\right\|=\operatorname{dist}\left(v_{t}, \mathscr{H}^{(0,0)}\right) \text {, for a unique } f_{v_{t}} \in \mathscr{H}^{(0,0)} .
$$

Assume that $v_{t, 1}+v_{t, 2}-v_{t}=\tilde{f}_{v_{t}} \in \mathscr{H}^{(0,0)}$ and that

$$
\begin{gathered}
\left\|v_{t}-g_{v_{t}}\right\|=\operatorname{dist}\left(v_{t}, \mathscr{H}^{(0,0)}\right) \\
\left\|v_{t, 1}+v_{t, 2}-\tilde{f}_{v_{t, 1}+v_{t 2}}\right\|=\operatorname{dist}\left(v_{t, 1}+v_{t, 2}, \mathscr{H}^{(0,0)}\right) .
\end{gathered}
$$

Therefore,

$$
\begin{aligned}
\left\|v_{t}-g_{v_{t}}\right\|=\| v_{t, 1} & +v_{t, 2}-\tilde{f}_{v_{t}}=g_{v_{t}} \|=\operatorname{dist}\left(v_{t}, \mathscr{H}^{(0,0)}\right) \\
& \geq \operatorname{dist}\left(v_{t, 1}+v_{t, 2}, \mathscr{H}^{(0,0)}\right)=\left\|v_{t, 1}+v_{t, 2}-\tilde{f}_{v_{t, 1}+v_{t, 2}}\right\| .
\end{aligned}
$$

On the other hand:

$$
\left\|v_{t, 1}+v_{t, 2}-f_{v_{t,}+v_{t, 2}}\right\|=\operatorname{dist}\left(v_{t, 1}+v_{t, 2}, \mathscr{H}^{(0,0)}\right)
$$




$$
=\left\|v_{t}+\tilde{f}_{v_{t}}-f_{v_{t 1}+v_{t 2}}\right\| \geq \operatorname{dist}\left(v_{t}, \mathscr{H}^{(0,0)}\right) .
$$

Thus $(\alpha)$ and $(\beta)$ imply that $v_{t, 1}+v_{t, 2}$ and $v_{t}$ are equidistant from $\mathscr{H}^{(0,0)}$. Next, taking into account that $v_{t}$ is in the orthogonal complement of $\mathscr{H}^{(0,0)}$, we deduce:

$$
\begin{gathered}
\left\|v_{t}\right\|^{2}=\left\|v_{t}-f_{v_{t}}\right\|^{2}+\left\|f_{v_{t}}\right\|^{2}=\left\|v_{t, 1}+v_{t, 2}+\tilde{f}_{v_{t}}\right\|^{2}, \\
\left\|v_{t, 1}+v_{t, 2}\right\|^{2}=\left\|v_{t, 1}+v_{t, 2}-f_{v_{t 1}+v_{t 2}}\right\|^{2}+\left\|f_{v_{t 1}+v_{t 2}}\right\|^{2} .
\end{gathered}
$$

Combining these, we deduce that $\left\|v_{t, 1}+v_{t, 2}\right\|^{2}+\left\|\tilde{f}_{v_{t}}\right\|^{2}=\operatorname{dist}+\left\|f_{v_{t}}\right\|^{2}$ and $\left\|f_{v_{t}}\right\|^{2}-\left\|\tilde{f}_{v_{t}}\right\|^{2}=\left\|f_{v_{t, 1}+v_{t 2}}\right\|^{2}$. Therefore $\left\|f_{v_{t}}\right\|^{2} \geq\left\|f_{v_{t 1}+v_{t 2}}\right\|^{2}$. Similarly,

$$
\begin{aligned}
& \left\|v_{t}+\tilde{f}_{v_{t}}\right\|^{2}=\operatorname{dist}+\left\|f_{v_{t 1}+v_{t 2}}\right\|^{2} \\
& \left\|v_{t}+\tilde{f}_{v_{t}}\right\|^{2}=\left\|v_{t}\right\|^{2}+\left\|\tilde{f}_{v_{t}}\right\|^{2}=\operatorname{dist}+\left\|f_{v_{t}}\right\|^{2}+\left\|\tilde{f}_{v_{t}}\right\|^{2} .
\end{aligned}
$$

Therefore $\left\|f_{v_{t, 1}+v_{t, 2}}\right\|^{2} \geq\left\|f_{v_{t}}\right\|^{2}$. Hence $\left\|f_{v_{t 1}+v_{t} 2}\right\|^{2}=\left\|f_{v_{t}}\right\|^{2}$. Therefore, we have

$$
f_{v_{t}}(\xi)=c f_{v_{t 1}+v_{t 2}}(\xi),(\xi) \in \mathbf{C}^{n}, c \in \mathbf{C},|c|=1
$$

But

$$
\begin{aligned}
\left\|v_{t}-f_{v_{t}}\right\|^{2} & =\left\|v_{t}-c f_{v_{t 1}+v_{t 2}}\right\|^{2} \\
& =\left\langle v_{t}-c f_{v_{t 1}+v_{t 2}}, v_{t}-c f_{v_{t 1}+v_{t 2}}\right\rangle \\
& =\left\|f_{v_{t}}\right\|^{2}+\left\|c f_{v_{t 1}+v_{t 2} 2}\right\|^{2} \\
& =\left\|f_{v_{t}}\right\|^{2}+\left\|f_{v_{t 1}+v_{t 2}}\right\|^{2} \\
& =\left\|v_{t}-f_{v_{t 1}+v_{t 2}}\right\|^{2} .
\end{aligned}
$$

Thus, $f_{v_{t}}=f_{v_{t 1}+v_{t 2}}$. This shows that $\left\|\tilde{f}_{v_{t}}\right\|^{2}=0$. Hence $\left|\tilde{f}_{v_{t}}\right|^{2}=0$ a.e which implies $\tilde{f}_{v_{t}}=0$. Hence we obtain the required identity $v_{t, 1}+v_{t, 2}=v_{t}$. This completes the proof of the theorem.

As an application of this theorem we get the following corollary:

Corollary 3.3. Let $f(t, \xi), f_{1}(t, \xi)$ be as above. Assume that there exist $K \subset L$, two compact convex subsets of $\mathbf{C}^{n}$ satisfying the following condition:

$$
H_{L}(\xi) \leq H_{K}(\xi)+o(\xi) \text { whenever } f_{t_{0}}(\xi)=0 .
$$

Then for every entire function $f_{1}(t, \xi)=f_{1, t}(\xi)$, satisfying the following uniformity condition for any $t \in U$ : 


$$
\log \left|f_{1, t}(\xi)\right| \leq H_{L}(\xi)+o(\xi) \quad \xi \in \mathbf{C}^{n}
$$

and for every $\varepsilon>0$, there exist two entire functions $f_{2, t}(\xi)=f_{2}(t, \xi), \tilde{f}_{3, t}(\xi)$ $=\tilde{f}_{3}(t, \xi)$ such that

$$
f_{1}(t, \xi)=f(t, \xi) f_{2}(t, \xi)+\tilde{f}_{3}(t, \xi)
$$

where $f_{2}$ satisfies

$$
\log \left|f_{2, t}(\xi)\right| \leq H_{L(\varepsilon)}(\xi)+o(\xi), \forall \xi \in \mathbf{C}^{n}
$$

and the remainder $\tilde{f}_{3}$ contains two terms: the term $f_{3}$ dominated by the support function of a $\varepsilon$-neighborhood $K$ and a term dominated by $\left|t-t_{0}\right|$, where $L(\varepsilon), K(\varepsilon)$ are $\varepsilon$-neighborhoods of $L, K$ respectively.

Proof. For $t_{0}$ fixed, then by previous theorem we have

$$
\begin{aligned}
f_{1}(t, \xi) & =f\left(t_{0}, \xi\right) f_{2}(t, \xi)+f_{3}(t, \xi) \\
& =\left[f\left(t_{0}, \xi\right)+f(t, \xi)-f(t, \xi)\right] f_{2}(t, \xi)+f_{3}(t, \xi) \\
& =f(t, \xi) f_{2}(t, \xi)+\left[f\left(t_{0}, \xi\right)-f(t, \xi)+f_{3}(t, \xi)\right] .
\end{aligned}
$$

Set $\tilde{f}_{3}(t, \xi)=\left[\left(f\left(t_{0}, \xi\right)-f(t, \xi)\right) f_{2}(t, \xi)+f_{3}(t, \xi)\right]$. Then $\log \left|f_{3, t}(\xi)\right| \leq H_{K(\varepsilon)}(\xi)$ $+o(\xi)$ by the previous theorem. And in addition we observe directly $\left(f\left(t_{0}, \xi\right)\right.$ $\left.-f(t, \xi)) f_{2}(t, \xi)\right)$ is dominated by $\left|t-t_{0}\right|$.

\section{§4. The Theorem of A. Sebbar-T. Aoki}

The following two theorems are generalizations of the original theorems of A. Sebbar. Since the weakening of a hypothesis is a direct consequence only of the previous division theorem, the proofs remain basically the same, but for the completeness of the argument they will be given here together with the new statements.

Theorem 4.1. Let $f$ be an entire function $\mathbf{C}^{n} \rightarrow \mathbf{C}$ of exponential type zero and let $Z_{f}$ be the set of its zeroes. If $U$ is an open convex subset of $\mathbf{C}^{n}$, then any solution $u$ of $f(D) u=0$ holomorphic in $U$ can be continued to a solution $v$ of $f(D) u=0$ holomorphic in $\Gamma_{\alpha\left(Z_{f}\right)}(U)=\Theta_{z_{f}}(U)$.

Proof. Let $\mathscr{H}_{f(D)}(U)$ be the space of the solutions $u$ of $f(D) u=0$ holomorphic in $U$, equipped with the topology induced by the $\mathscr{H}(U)$. Put $V=\Theta_{Z_{f}}(U)$, then we are going to show that the restriction map

$$
R: \mathscr{H}_{f(D)}(V) \mapsto \mathscr{H}_{f(D)}(U)
$$


is surjective. Since the exponential polynomial solutions are dense in both spaces, $\operatorname{Im} R$ is dense. Therefore the surjectivity is equivalent to

$$
{ }^{t} R: \mathscr{H}_{f(D)}^{\prime}(U) \mapsto \mathscr{H}_{f(D)}^{\prime}(V)
$$

is surjective, because ${ }^{t} R$ is already injective. Let $T_{1} \in \mathscr{H}_{f(D)}^{\prime}(V)$, which can be considered as an analytic functional on $V$. By the theorem of EhrenpreisMartineau there exists a compact convex subset $L$ of $V$ so that:

$$
\log \left|\hat{T}_{1}(\zeta)\right| \leq H_{L}(\zeta)+\alpha_{1}, \zeta \in \mathbf{C}^{n}
$$

where $\alpha_{1}$ is a positive constant and $\hat{T}_{1}$ is the Fourier-Borel transform of $T_{1}$ :

$$
\hat{T}_{1}(\zeta)=\left\langle T_{1}, \exp (\langle z, \zeta\rangle)\right\rangle, \zeta \in \mathbf{C}^{n}
$$

Since $L$ is contained in $V=\bigcup_{K \in \pi^{\prime}(U)}{\overline{\left(\Theta_{W_{f}}(K)\right.}}^{\circ}$, there exists $K$ a compact, convex subset of $U$ so that

$$
L \subset \Theta_{Z_{f}}(K) \subset \bigcup_{r \geq 0} \Theta_{Z_{f}}(K),
$$

and we have therefore, for some $r \geq 0, L \subset \Theta_{Z_{f, r}}(K)$. Hence

$$
H_{L}(\zeta) \leq H_{K}(\zeta)+r, \zeta \in Z_{f}
$$

where we may assume that $K \subset L$, otherwise we replace $L$ by $c v(K \cup L)$, the convex hull of $K \cup L$. By the division theorem proved above, for any $\varepsilon>0$, in particular for $\varepsilon_{0}>0$ small enough so that $K_{\varepsilon_{0}} \subset U$ and $L_{\varepsilon_{0}} \subset V$ there exist entire functions $f_{2}$ and $f_{3}$ so that $\hat{T}_{1}=f f_{2}+f_{3}$, where

$$
\begin{aligned}
& \log \left|f_{2}(\zeta)\right| \leq H_{L_{\varepsilon_{0}}}(\zeta)+a_{2}\left(\varepsilon_{0}\right) \log (2+|\zeta|), \zeta \in \mathbf{C}^{n}, \\
& \log \left|f_{3}(\zeta)\right| \leq H_{K_{\varepsilon_{0}}}(\zeta)+a_{3}\left(\varepsilon_{0}\right) \log (2+|\zeta|), \zeta \in \mathbf{C}^{n} .
\end{aligned}
$$

Again by the theorem of Martineau-Ehrenpreis there exist $T_{2} \in \mathscr{H}_{f(D)}^{\prime}(U)$ and $T_{3} \in \mathscr{H}_{f(D)}^{\prime}(V)$ so that: $f_{2}=\hat{T}_{2}, f_{3}=\hat{T}_{3}$. For every $v \in \mathscr{H}_{f(D)}(V)$ we then have:

$$
\left\langle T_{1}, v\right\rangle=\left\langle T_{2}, f(D) v\right\rangle+\left\langle T_{3},\left.v\right|_{U}\right\rangle
$$

Let $S \in \mathscr{H}_{f(D)}^{\prime}(U)$ be the restriction of $T_{3}$ to the $\mathscr{H}_{f(D)}(U)$. For every $v \in \mathscr{H}_{f(D)}(V)$ we deduce

$$
\left\langle{ }^{t} R(S), v\right\rangle=\langle S, R(v)\rangle=\left\langle T_{3},\left.v\right|_{U}\right\rangle=\left\langle T_{1}, v\right\rangle
$$

which implies the desire surjectivity. 
Theorem 4.2 (A. Sebbar-T. Aoki). Let L be a differential operator of infinite order with constant coefficients. Let $U$ be an open convex set in $\mathbb{C}^{n}$ and $U^{\prime}$ be the polar envelope of $U$ with respect to the characteristic set $\alpha(C h(L))$ of L. Suppose that $u$ is holomorphic in $U$ and $L u$ has an analytic continuation to $U^{\prime}$. Then $u$ has analytic continuation to $U^{\prime}$.

Proof. We have $L=f(D)$, where $f$ is an entire function of exponential type zero and $V=U^{\prime}=\Theta_{z_{f}}(U)$. We want to show that if $u \in \mathscr{H}(U)$ and $v \in \mathscr{H}(V), L u=\left.v\right|_{U}$, then $u$ can be analytically continued to a solution $u_{0}$ of $L u_{0}=v$ holomorphic in $V$. Actually, since the operator $L: \mathscr{H}(V) \mapsto \mathscr{H}(V)$ is surjective there exists $v_{0} \in \mathscr{H}(V)$ so that $L v_{0}=v$ which shows that $u-\left.v_{0}\right|_{U}$ is a holomorphic solution in $U$ of $L u=0$. By the Theorem 4.1 there exists $\tilde{u}$, holomorphic solution of $L u=0$ in $V$, so that $\tilde{u} \mid U=u-v_{0}$. The function $\tilde{u}+v_{0}$ is holomorphic in $V$, it is the analytic continuation of $u$ and satisfies:

$$
L\left(\tilde{u}+v_{0}\right)=L \tilde{u}+L v_{0}=L v_{0}=v
$$

\section{Acknowledgement}

The authors would like to thank the referee and Prof. Mitsuo Morimoto for their very helpful sugges tions. A. Vidras would like to express his gratitude to Prof. T.Kawai and RIMS for their warm hospitality.

\section{References}

[1] Aoki, T., Calcul exponentiel des opérateurs microdifférentiels d'ordre infini I, Ann. Inst. Fourier (Grenoble), 33 (1983), 227-250.

[2] Calcul exponentiel des opérateurs microdifférentiels d'ordre infini II, Ann. Inst. Fourier (Grenoble), 36(1986), 143-165.

[3] , Existence and continuation of holomorphic solutions of differential equations of infinite order. Advances in Math. , (72). 1988, 261-283.

[4] Berenstein, C.A. and Gay, R., Complex analysis, an introduction to harmonic analysis, SpringerVerlag. (to appear)

[5] Berenstein, C.A. and Taylor, B.A., Interpolation problems in $\mathbb{C}^{n}$ with applications to harmonic analy sis. Journal Analyse Math ., (38 )1980, 188-254.

[6] Boas, R., Entire Functions, Academic Press, 1954.

[7] Braun, R.W., Meise, R and Taylor, B.A., Ultradifferentiable functions and Fourier analysis, Resultate Math ., 17 (1990), 206-237.

[8] Ehrenpreis, L., Fourier analysis in Several Complex Variables. Wiley, 1970.

[9] Hörmander, L., An Introduction to Complex Analysis in Several Complex Variables, North -Holland, 1990.

[10] Kashiwara, M. and Shapira, P., Micro-hyperbolic systems, Acta Math., 142 (1979), 1-55. 
[11] Kawai, T., On the theory of Fourier hyperfunctions and its applications to partial differential * equations with constant coefficients, Jour. Fac. Sci. Univ. Tokyo, 17 (1970), 467-517.

[12] Kiselman, C.O., Prolongement de solutions d'une équation aux dérivées partielles á coefficiets constants, Bull. Soc. Math. Fr., 97 (1969), 329-356.

[13] - On unique supports of analytic functionals. Arkiv för Mat., 6 (1965), 307-318.

[14] Levin, B. Ja., Distribution of zeroes of entire functions, AMS Publ., 1964.

[15] Sebbar, A., Prolongement des solutions holomorphes de certain opérateurs d'ordre infini a coefficients constants, Séminaire P. Lelong-H. Skoda (Analyse), 1978-79, Springer-Verlag. 
